\title{
Contact and Symplectic Topology: Mastering the Art of Front Cooking
}

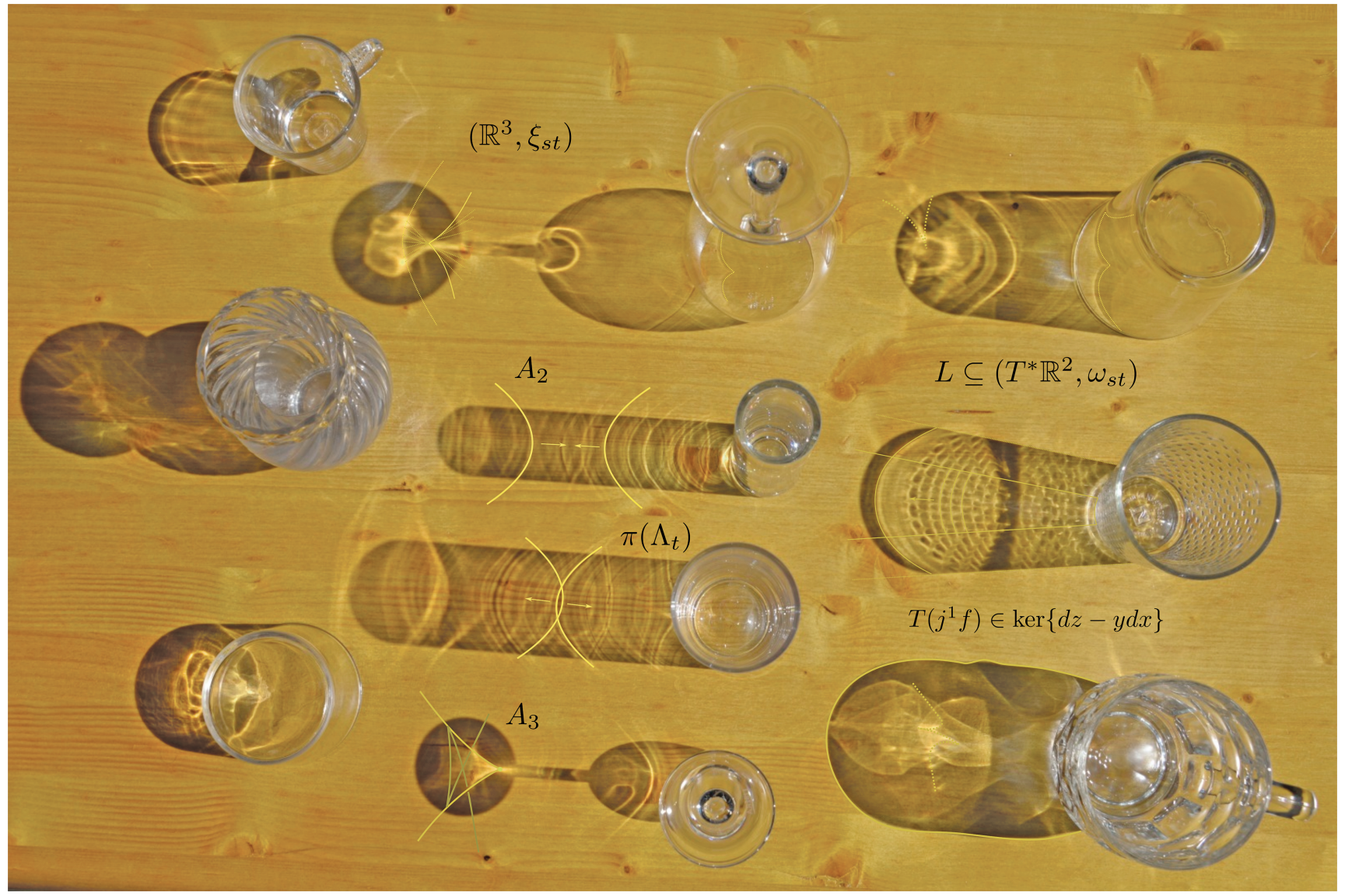

\section{Roger Casals}

\section{A Bird's Eye View}

Classification results are relevant in many areas of mathematics. The initial data is often a set of objects and an isomorphism relation-we invite the reader to think of their favorite classification problem. A few examples are:

Roger Casals is a professor of mathematics at the University of California Davis. His email address is casals@ucdavis.edu.

For permission to reprint this article, please contact:

reprint-permission@ams.org.

DOI: https://doi.org/10.1090/noti2276 (i) knots $K \subseteq \mathbb{R}^{3}$, classified up to smooth isotopy, or smooth manifolds up to diffeomorphism,

(ii) metric spaces, e.g., Riemannian manifolds, understood up to isometries or quasi-isometries,

(iii) discrete groups, up to (virtual) isomorphism, or Lie groups and their Lie algebras,

(iv) the classification of integer quadratic forms, classified up to classes or genera-or the solutions to a Diophantine equation up to descent,

(v) the (space of) functions solving a partial differential equation, solutions possibly considered up to compactly supported smooth functions or gauge equivalence. 
Classification problems aim to either prove two given objects $X_{1}$ and $X_{2}$ are isomorphic, $X_{1} \cong X_{2}$, or that they are not isomorphic. In the former case, an isomorphism must be constructed, either explicitly or by abstract means. In the latter, one must argue such an equality cannot exist; this is frequently achieved by constructing and computing an invariant $I(X)$, associated to each object $X$.
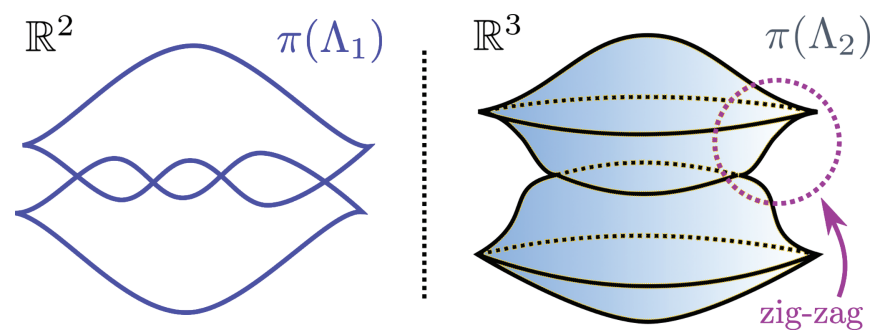

Figure 1. Legendrian wavefront $\pi\left(\Lambda_{1}\right) \subseteq \mathbb{R}^{2}$ for a (2,4)-torus link $\Lambda_{1} \subseteq \mathbb{R}^{3}$ (left). Legendrian wavefront $\pi\left(\Lambda_{2}\right) \subseteq \mathbb{R}^{3}$ for a 2-sphere $\Lambda_{2} \subseteq \mathbb{R}^{5}$ (right).

This invariant $I$ could be anything: a number, a polynomial, an Abelian group, a category, ${ }^{1}$ a topological space, or a kind of potato-as long as it satisfies that $I\left(X_{1}\right) \cong I\left(X_{2}\right)$ if $X_{1} \cong X_{2}$, it can be used to argue that $X_{1} \not X_{2}$ by showing that $I\left(X_{1}\right) \not I\left(X_{2}\right)$.

Examples. Instances of invariants include the dimension of a vector space, the index of Fredholm operator, the class number of a number field, the genus of a surface, curvatures in Riemannian manifolds, the Jones polynomial of a knot, the Poincaré polynomial of a space, the homology groups of a space, or the category of coherent sheaves on an algebraic variety.

In general, one still has to show $I\left(X_{1}\right) \nRightarrow I\left(X_{2}\right)$, which is also a classification problem-the trick is to choose invariants $I$ which are easily distinguished: most mathematicians would be comfortable telling numbers, polynomials, and Abelian groups apart, and such invariants are most frequently used (categories, spaces, and potatoes might have a more niche crowd).

The field of contact and symplectic topology $[1,6]$ has seen developments on both sides of the aisle: new invariants $I$ distinguishing objects, and new techniques to construct isomorphisms.

\section{Developments: Two New Directions}

This article will introduce some of the objects studied in contact and symplectic topology and discuss some of the results that have been proved. ${ }^{2}$ We focus on two particular

\footnotetext{
${ }_{1}^{1}$ E.g., "computing a category" might mean finding a set of generators, or presenting it as the differential graded derived (dg-derived) category of modules over an algebra we understand.

${ }^{2}$ The four main protagonists (contact, symplectic, Legendrian, and Lagrangian) will be defined momentarily and the reader is welcome to skip to the next section if needed.
}

developments:

(1) The discovery of a flexible class of contact structures and symplectic structures.

(2) The study of Legendrian and Lagrangian submanifolds using microlocal sheaf theory.

In these results, Legendrian wavefronts, which are certain singular hypersurfaces $\pi(\Lambda) \subseteq \mathbb{R}^{n}$, have played a crucial role; see Figure 1. These will be discussed in depth in the upcoming section. The title of this article is a reference to Mastering the Art of French Cooking. For us, it is the study of (wave)front diagrams, with all its flavors and different recipes, that allows us to prove new results and enjoy some of the wonders of our mathematical kitchen.

Example. For a Legendrian knot $\Lambda \subseteq\left(\mathbb{R}^{3}, \xi_{\text {st }}\right)$, the front $\pi(\Lambda) \subseteq \mathbb{R}^{2}$ is akin to a planar knot diagram, except it allows for cusps and all planar crossings are overcrossings; ${ }^{3}$ see Figure 1 (left) and Figure 4. For a Legendrian surface $\Lambda \subseteq$ $\left(\mathbb{R}^{5}, \xi_{\text {st }}\right)$, the front $\pi(\Lambda) \subseteq \mathbb{R}^{3}$ is a singular surface in $\mathbb{R}^{3}$, as in Figure 1 (right) or the blue flying saucer in Figure 2. Note that we are drawing surfaces in 5-dimensions by drawing fronts in 3-space.

Given a Legendrian wavefront $\pi(\Lambda)$, we will build a symplectic manifold $W(\Lambda)$ and two contact manifolds $Y_{ \pm 1}(\Lambda)$, and also construct invariants. Figure 2 schematically shows these possibilities.

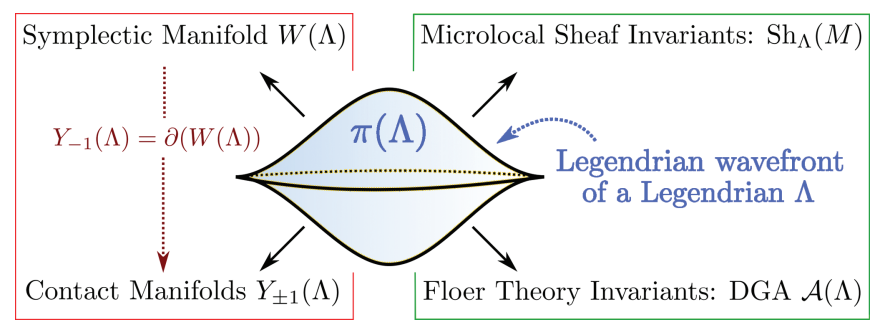

Figure 2. A Legendrian wavefront $\pi(\Lambda)$, from which we can build geometric manifolds (left) or compute algebraic invariants (right). $M$ is the smooth manifold where the wavefront lives, typically $M=\mathbb{R}^{n}$.

Part (1) above belongs to the realm of $h$-principles [12]. The theory of $h$-principles is constructive, a tenet being that "if certain algebraic obstructions vanish, then a geometric construction is possible." (In broad terms, $h$-principles are theorems where "algebra implies geometry.") In our context, $h$-principles are results allowing one to conclude that, for certain classes of objects and (actually computable) invariants $I$, the equality $I\left(X_{1}\right) \cong I\left(X_{2}\right)$ implies that $X_{1} \cong X_{2}$. By definition, $X_{1}, X_{2}$ are flexible with respect to an invariant $I$ when $I\left(X_{1}\right) \cong I\left(X_{2}\right)$ implies $X_{1} \cong X_{2}$. Thus, should these flexible objects exist, the invariant $I$ gives a complete classification! For a given invariant $I$, it is interesting to study

\footnotetext{
${ }^{3}$ Northwest-Southeast strand above Southwest-Northeast.
} 
whether a nonempty class of flexible objects exists and, if so, decide whether a given object is flexible.

In contact and symplectic topology, these flexible classes are defined by the existence of a local zig-zag in Legendrian wavefronts; ${ }^{4}$ such a zig-zag is shown in Figure 1 (right). A wavefront with a zig-zag is said to be stabilized. The following result $[2,5,6]$ summarizes ${ }^{5}$ three flexible developments.

Theorem 1. In higher dimensions, i.e., $\operatorname{dim}(\Lambda) \geq 2$ for Legendrians and $\operatorname{dim}(W) \geq 6$ for symplectic manifolds, the following hold:

(i) Let $\Lambda_{1}, \Lambda_{2} \subseteq(Y, \xi)$ be smoothly isotopic Legendrian submanifolds, each admitting a stabilized wavefront. Then $\Lambda_{1}$ is Legendrian isotopic to $\Lambda_{2}$.

(ii) Let $W_{1}, W_{2}$ be diffeomorphic symplectic manifolds, each admitting a stabilized front handlebody. Then $W_{1}$ is symplectic isomorphic to $W_{2}$.

(iii) Let $Y_{1}, Y_{2}$ be diffeomorphic contact manifolds, each obtained by $(+1)$-surgery on a stabilized wavefront. Then $Y_{1}$ is contact isomorphic to $Y_{2}$.

In fact, we also know several equivalent characterizations [5] of the hypothesis in Theorem 1(iii), e.g., in terms of contact open books and the existence of overtwisted disks.

Each of the three statements in Theorem 1 is of the following form: we have two objects $X_{1}, X_{2}$ which we want to compare. Then if both $X_{1}, X_{2}$ verify a certain topological property (being diffeomorphic and "stabilized"), then $X_{1} \cong X_{2}$. The notions featured in Theorem 1 will be explained shortly. The message is that, in contact and symplectic topology, we now have verifiable local properties (of a wavefront) which globally characterize certain isomorphism classes of geometric objects, i.e., if you see a zig-zag somewhere in your wavefront diagram $\pi(\Lambda)$, this characterizes completely the symplectic and contact topology of $\Lambda, W(\Lambda)$, and $Y_{ \pm 1}(\Lambda)$ given the underlying smooth data. (This is rather exclusive to contact and symplectic topology: e.g., for a knot diagram or a Riemannian manifold, no local property near one point will typically be able to globally characterize the knot or the Riemannian metric.)

In contrast, part (2) above has provided new invariants to help the classification and study of contact and symplectic structures. ${ }^{6}$ This relies on new and fruitful connections to cluster algebras $[3,17]$, differential equations, and

\footnotetext{
${ }^{4}$ The importance of zig-zags in h-principles, and in general wrinkled maps, goes back to Eliashberg-Mishachev (1997).

${ }^{5}$ For notational simplicity, we write smooth isotopy for a smooth isotopy which includes the corresponding formal data required by the h-principle; the same for diffeomorphisms.

${ }^{6}$ Floer-theoretic invariants date back to the 1980 s, the dg-algebras $\mathcal{A}$ being developed in the 2000s. The resurgence of microlocal sheaf theory belongs to the last decade 2010-20.
}

homological mirror symmetry. A class of invariants that are shaping the field is given by studying categories of constructible sheaves, with appropriate constraints [13-15].

Let $M$ be a smooth manifold. These sheaves are intuitively given by assigning a finite-dimensional vector space at each point of $M$. The dimensions of the vector spaces might potentially jump as we vary the point. Legendrian wavefronts $\pi(\Lambda) \subseteq M$ serve as constraint: we only allow these dimensional jumps to happen when we cross the wavefront. This is morally what being constructible with respect to a wavefront means; the set of such sheaves is denoted by $\operatorname{Sh}_{\Lambda}(M)$. In precise terms, let $T^{\infty} M$ be a unit cotangent bundle. Then, we have the following.

Theorem 2 ([13, 14, 18]). The category $\operatorname{Sh}_{\Lambda}(M)$ of constructible sheaves microlocally supported at a Legendrian $\Lambda \subseteq$ $T^{\infty} M$ is a Legendrian invariant $I(\Lambda)$. Furthermore, it can often be computed combinatorially by using a wavefront for $\Lambda$.

The computability is a strong virtue of such categories $\mathrm{Sh}_{\Lambda}(M)$. The result above is remarkably efficient at distinguishing Legendrian submanifolds $\Lambda_{1}, \Lambda_{2} \subseteq T^{\infty} M$ which are smoothly isotopic but not Legendrian isotopic. Many interesting results in the field have been discovered or reproved thanks to the use of microlocal sheaf theory [1315]. Two new examples $[3,17]$ are given in the following theorem.

Theorem 3. (i) The moduli space of Lagrangian fillings of positive Legendrian links $\Lambda \subseteq\left(\mathbb{R}^{3}, \xi_{\text {st }}\right)$ admits a (partial) cluster structure.

(ii) The Legendrian (max-tb) representatives of $(n, m)$ torus links, $n \geq 3, m \geq 6$, admit infinitely many distinct Lagrangian fillings. In fact, most max-tb Legendrian representatives of a positive braid admit infinitely many distinct Lagrangian fillings.

The Hamiltonian isotopy class of an embedded exact Lagrangian filling specifies a cluster chart in the moduli space of Theorem 3(i), and it is shown that these cluster charts can be used to distinguish exact Lagrangian fillings as in Theorem 3(ii). ${ }^{7}$

\section{Contact and Symplectic Topology}

Let us first introduce the four main characters in the field: symplectic and contact structures, and Legendrian and Lagrangian submanifolds. In this article, the symplectic and Lagrangian dimensions will be $2 n$ and $n$, and the contact and Legendrian dimensions will be $2 n-1$ and $n-1$, respectively.

Definition 1. A symplectic structure $(X, \omega)$ on a real $2 n$ dimensional smooth manifold $X$ is the choice of a nondegenerate closed 2-form $\omega$ on $X$.

\footnotetext{
${ }^{7}$ This relates to the wall-crossing phenomena present in Morse theory and mirror symmetry.
} 
In practice, the integral $\int_{S} \omega \in \mathbb{R}$ over a 2-dimensional surface $S$ generalizes the notion of 2-dimensional area to any surface $S \subseteq X$. In contrast to a Riemannian metric, the 2 -form $\omega$ is antisymmetric and perceives orientations. Thus, nonpositive (symplectic) areas are allowed. Note that the three infinite families of simple Lie algebras are $\mathfrak{S l}_{n}$, corresponding to volume-preserving geometry, $\mathfrak{S}_{n_{n}}$, giving (pseudo)Riemannian geometry, and $\mathfrak{s p}_{n^{\prime}}$ which yields symplectic geometry.

Example. Consider the $2 n$-dimensional ball

$$
\mathbb{D}^{2 n}:=\left\{(x, y) \in \mathbb{R}^{n} \times \mathbb{R}^{n}:\|x\|^{2}+\|y\|^{2} \leq 1\right\} .
$$

Then $\omega_{\mathrm{st}}:=d x_{1} \wedge d y_{1}+\cdots+d x_{n} \wedge d y_{n}$ is a symplectic form. Given an embedded surface $S \subseteq \mathbb{R}^{2 n}$, the real number $\int_{S} \omega_{\text {st }} \in \mathbb{R}$ is obtained as follows: for each $i \in[1, n]$, project $S$ to the 2-plane $\left\langle x_{i}, y_{i}\right\rangle \subseteq \mathbb{R}^{2 n}$ and compute the signed area $A_{i}$ of this image. Then $\int_{S} \omega_{\text {st }} \in \mathbb{R}$ is the sum $A_{1}+\cdots+A_{n}$. See Figure 3 and note that some, even all, of the $A_{i}$ might be zero, e.g., the 2-torus $L:=\left\{x_{1}^{2}+y_{1}^{2}=\right.$ $\left.1, x_{2}^{2}+y_{2}^{2}=1, x_{j}=y_{j}=0,2<j \leq n\right\} \subseteq \mathbb{R}^{2 n}$ has all $A_{i}=0$.

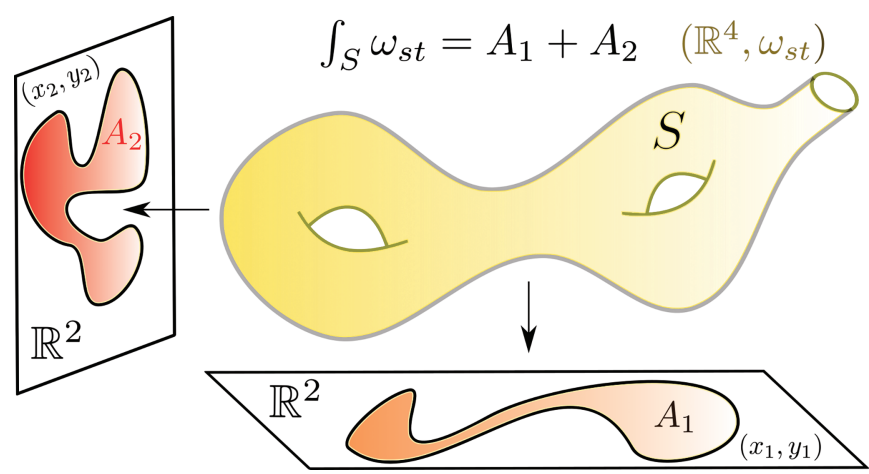

Figure 3. A surface $S \subseteq \mathbb{R}^{4}$ and the number $\int_{S} \omega_{\text {st }}$ given by the symplectic structure.

Symplectic structures are abundant: complex projective manifolds, or any manifold $X=\left\{p_{1}=\cdots=p_{n}=\right.$ $0\} \subseteq \mathbb{C}^{N}$ cut out by complex polynomials, are symplectic. The phase space of any physical system on a configuration space $M$, i.e., the cotangent bundle $\left(T^{*} M, \lambda_{\text {can }}\right)$ and its reductions, also admits symplectic structures. These come from the 2 -from $d \lambda_{\text {can }}$, where $\lambda_{\text {can }}$ is the canonical Liouville 1-form on $T^{*} M$. Most interesting aspects of symplectic topology are global: near a point $p \in(X, \omega)$, the symplectic structure is always given by the above example $\left(\mathbb{D}^{2 n}, \omega_{\mathrm{st}}\right)[1]$.

Definition 2. A contact structure on a real $(2 n-1)$ dimensional smooth manifold $Y$ is a (locally) generic ${ }^{8}$ and

${ }^{8 \text { The precise condition is "maximally nonintegrable"; by the Frobenius Integra- }}$ bility Theorem, this is equivalent to the algebraic condition $\xi=\operatorname{ker}(\alpha)$ and $\alpha \wedge(d \alpha)^{n-1} \neq 0$, where $\alpha$ is a 1 -form in $Y$. smooth choice of hyperplane $\xi_{y} \subseteq T_{y} Y$ at each tangent space.

Example. Consider the sphere $\mathbb{S}^{2 n-1}=\partial \mathbb{D}^{2 n}$. The kernel $\xi_{\text {st }}:=\operatorname{ker}\left(\lambda_{\mathrm{st}}\right)$ is a contact structure, where $\lambda_{\mathrm{st}}$ is the restriction of the 1 -form $\lambda_{\text {st }}:=\frac{1}{2} \sum_{i=1}^{n}\left(x_{i} d y_{i}-y_{i} d x_{i}\right)$ to the boundary $\partial \mathbb{D}^{2 n}$. Removing a point from the sphere yields a contact structure $\left(\mathbb{R}^{2 n-1}, \xi_{\text {st }}\right)$.

Contact structures are also bountiful. In many cases, the intersection $Y=X \cap \mathbb{S}^{2 N-1}$ of a manifold $X=\left\{p_{1}=\cdots=\right.$ $\left.p_{n}=0\right\} \subseteq \mathbb{C}^{N}=\mathbb{R}^{N} \oplus i \mathbb{R}^{N}$ with the unit sphere $\mathbb{S}^{2 N-1} \subseteq$ $\mathbb{C}^{N}$ has a canonical contact structure $\xi=T Y \cap i(T Y)$. Similarly, the energy level sets $T^{\infty} M$ of phase spaces and their reductions generically have contact structures coming from $\operatorname{ker}\left(\lambda_{\text {can }}\right)$. Contact topology is also global in nature: near a point $p \in(Y, \xi)$, the contact structure is given by the example $\left(\mathbb{R}^{2 n-1}, \xi_{\text {st }}\right)$ above [9].

Remark. É. Cartan classified all distributions $D \subseteq T Y$ that locally have a unique normal form. There are four classes: (i) nonvanishing vector fields, which lead to smooth dynamics, (ii) Engel structures, which are 4-dimensional, (iii) even-contact structures, and (iv) contact structures.

A salient relation between a symplectic manifold $(X, \omega)$ and a contact manifold $(Y, \xi)$ arises when $Y=\partial X$ is the boundary of $X$ and $\omega=d \lambda$ is the differential of a 1-form. Then the hyperplanes $\xi_{y}=\left\{v \in T_{y} Y: \lambda_{y}(v)=0\right\}$ are the linear subspaces $\operatorname{ker}(\lambda)$, and these often form a contact structure $(\partial X, \xi)$ if $\lambda$ is appropriately chosen. Note that the two examples $\left(\mathbb{S}^{2 n-1}, \xi_{\mathrm{st}}\right)=\partial\left(\mathbb{D}^{2 n}, \omega_{\mathrm{st}}\right)$ above fit into this framework as $d \lambda_{\text {st }}=\omega_{\text {st }}$. In general, it is fruitful to think of contact manifolds $(Y, \xi)=(\partial X, \operatorname{ker} \lambda)$ as boundaries of (exact) symplectic manifolds $(X, \omega)=(X, d \lambda){ }^{9}$

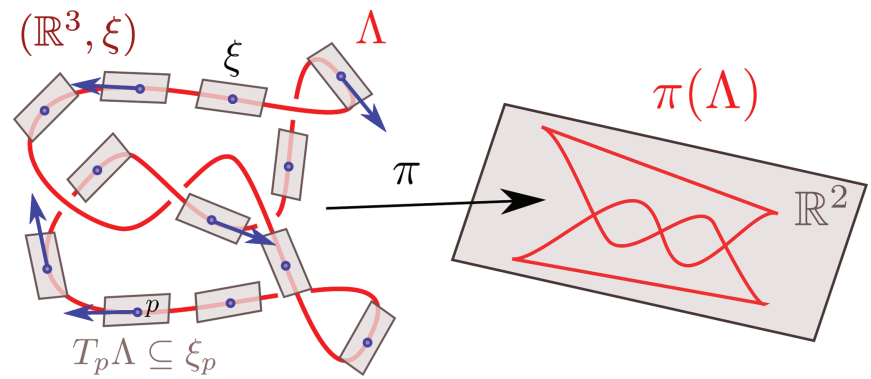

Figure 4. A Legendrian trefoil knot $\Lambda \subseteq \mathbb{R}^{3}$ and its wavefront $\pi(\Lambda) \subseteq \mathbb{R}^{2}$. Legendrian means that the tangent vectors to $\Lambda$ belong to the contact 2-plane $\xi$.

A Lagrangian submanifold $L \subseteq(X, \omega)$ is any submanifold such that the restriction $\left.\omega\right|_{L}=0$ is zero and

\footnotetext{
${ }^{9}$ This apparently innocent pairing is strengthened by a tenet in the field: "the contact boundary $(\partial X, \operatorname{ker} \lambda)$ knows about the symplectic interior $(X, d \lambda)$. " For instance, suppose that $(X, d \lambda)$ symplectically coincides with $\left(\mathbb{R}^{2 n}, \omega_{\mathrm{st}}\right)$ away from a compact set. Then $X$ must be diffeomorphic to $\mathbb{R}^{2 n}$, and even symplectic isomorphic to $\left(\mathbb{R}^{4}, \omega_{\mathrm{st}}\right)$ for $n=2$.
} 
$\operatorname{dim}(X)=2 \operatorname{dim}(L)$. On the contact side, an isotropic submanifold $\Lambda \subseteq(Y, \xi)$ is a submanifold of $Y$ such that at each point $p \in \Lambda$, the tangent space $T_{p} \Lambda$ is contained in the hyperplane $\xi_{p}$. An isotropic submanifold $\Lambda \subseteq(Y, \xi)$ is said to be Legendrian if $\operatorname{dim}(Y)=2 \operatorname{dim}(\Lambda)+1$.

In fact, $\left.\omega\right|_{L}=0$ implies the dimensional inequality $2 \operatorname{dim}(L) \leq \operatorname{dim}(X)$ and, similarly, $T \Lambda \subseteq \xi$ implies $2 \operatorname{dim}(\Lambda)+1 \leq \operatorname{dim}(Y)$. Thus, Lagrangian and Legendrian submanifolds have the maximal possible dimension given their defining (isotropic) constraints. Figure 4 (left) depicts a Legendrian knot $\Lambda \subseteq\left(\mathbb{R}^{3}, \operatorname{ker} \lambda\right)$.

Example. Lagrangian submanifolds $L \subseteq\left(\mathbb{R}^{2 n}, \omega_{\text {st }}\right)$ generalize the graphs $\operatorname{gr}(d f):=\{y=d f(x)\} \subseteq \mathbb{R}^{2 n}$ of the derivative $d f: \mathbb{R}^{n} \rightarrow \mathbb{R}^{n}$ of an $\mathbb{R}$-valued function $f=f(x) \in C^{\infty}\left(\mathbb{R}^{n}\right)$, which are indeed Lagrangian for $\omega_{\text {st }}$. In fact, any Lagrangian is locally of this form. Similarly, Legendrian submanifolds $\Lambda \subseteq\left(\mathbb{R}^{2 n-1}, \xi_{\text {st }}\right)$, with $\xi_{\text {st }}=\operatorname{ker}\left\{d z-y_{1} d x_{1}-\cdots-y_{n-1} d x_{n-1}\right\}$, generalize the 1-jet graphs $j^{1}(f):=\{(x, y, z): y=d f(x), z=f(x)\} \subseteq \mathbb{R}^{2 n-1}$ of $f \in C^{\infty}\left(\mathbb{R}^{n-1}\right)$, containing the information of the function $f$ and its derivatives $d f$. Any such $j^{1}(f)$ is Legendrian for $\xi_{\text {st }}$ and, conversely, any Legendrian is locally of this form.

The relation between $L$ and $\Lambda$ parallels that of $X$ and $\partial X$ : in many cases, the boundary $\partial L=L \cap \partial X$ of a properly embedded Lagrangian submanifold $L \subseteq(X, d \lambda)$ is a Legendrian submanifold $\Lambda=\partial L \subseteq(\partial X, d \lambda){ }^{10}$

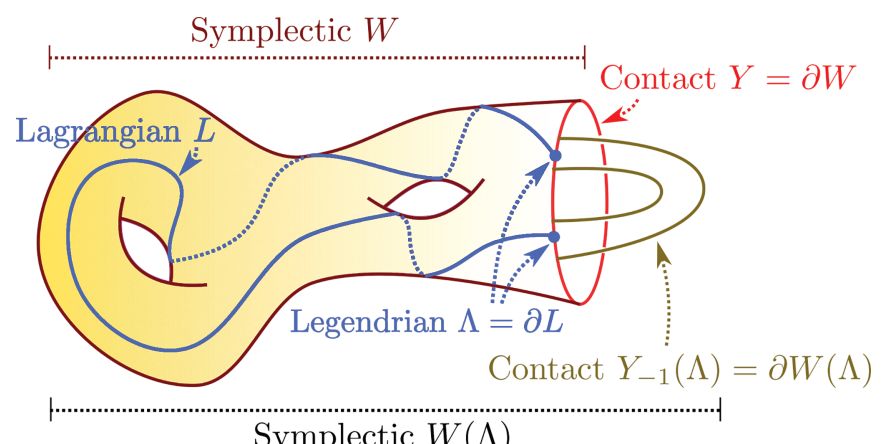

Figure 5. A symplectic manifold $W^{2 n}$ with contact boundary $Y^{2 n-1}=\partial W$, and a Lagrangian $L^{n} \subseteq W$ with Legendrian boundary $\Lambda^{n-1}=\partial L$.

Pictorial conclusion. Figure 5 depicts a rather general situation where the four main characters appear at once. Researchers in the field look at many different questions; including the classification of all symplectic (or contact) structures on a smooth manifold, as well as the

\footnotetext{
${ }^{10}$ The previous tenet also holds: "the Legendrian boundary $\Lambda=\partial L \subseteq$ $(\partial X, \operatorname{ker} \lambda)$ knows about the Lagrangian interior $L \subseteq(X, d \lambda)$. " E.g., if an embedded (exact) Lagrangian surface $L \subseteq\left(\mathbb{D}^{4}, \omega_{\mathrm{st}}\right)$ bounds a Legendrian knot $\Lambda=\partial L \subseteq \partial\left(\mathbb{D}^{4}, \omega_{\mathrm{st}}\right)$, then $L$ must have the minimal 4-genus of the knot $\Lambda \subseteq \mathbb{S}^{3}$.
}

classification of Lagrangian (or Legendrian) submanifolds. Theorems 1, 2, and 3 above help us classify some such structures.

\section{Studying Legendrians: Wavefronts}

Symplectic and contact structures are rather hard to visualize: one is a 2-form and the other a hyperplane distribution. We will be able to translate problems on contact and symplectic structures into Legendrians, which are often more easily visualized and manipulated. This leads to some key questions: e.g., given two Legendrian submanifolds $\Lambda_{1}, \Lambda_{2}$, how do we show $\Lambda_{1} \cong \Lambda_{2}$ or prove that $\Lambda_{1} \not \Lambda_{2}$ ?

At its core, the answer to this question is by drawing them: by picturing a Legendrian $\Lambda$ we will be able to both pin down properties of the drawing that might characterize the Legendrian isotopy class of $\Lambda$ and compute invariants $I(\Lambda)$ that distinguish Legendrians. Let us focus on $(Y, \xi)=\left(\mathbb{R}^{2 n-1}, \xi_{\mathrm{st}}\right)$, as this is the local model. The projection $\pi: \mathbb{R}^{2 n-1} \longrightarrow \mathbb{R}^{n}, \pi(x, y, z)=(x, z)$, has the following property: the image $\pi(\Lambda) \subseteq \mathbb{R}^{n}$ of any Legendrian recovers the Legendrian $\Lambda \subseteq\left(\mathbb{R}^{2 n-1}, \xi_{\text {st }}\right)$ by setting the $y_{i^{-}}$ coordinates to be the $x_{i}$-slope of the tangent plane of $\pi(\Lambda)$, i.e., $y_{i}:=\partial_{x_{i}} z\left(x_{1}, \ldots, x_{n-1}\right)$. Indeed, this is just the analytic incarnation of the condition $T \Lambda \subseteq \xi_{\text {st }}$.

The image $\pi(\Lambda) \subseteq \mathbb{R}^{n}$ is known as the wavefront of $\Lambda$. Figures 1, 2, and 4 depict examples of wavefronts. The wavefront $\pi(\Lambda)$ is typically a singular hypersurface, despite $\Lambda$ being embedded: the singularities acquired by $\pi(\Lambda)$ come from the singularities of the restricted projection $\left.\pi\right|_{\Lambda}$. Hence, we can study Legendrians in $\left(\mathbb{R}^{2 n-1}, \xi_{\text {st }}\right)$ by studying certain singular hypersurfaces in $\mathbb{R}^{n}$. Building on the theory of singularities [1], there is a diagrammatic calculus for wavefronts $[1,4,11]$. In the same way that one may tackle knots through their diagrams, and manipulate smooth 4-manifolds with Kirby calculus, contact and symplectic topologists can manipulate Legendrians and Lagrangians using wavefront diagrams.

\section{Two Applications}

Sample problems that we can now address with contact and symplectic techniques include the following two problems.

Problem 1 (Affine varieties). Let us consider the two symplectic manifolds $W_{1}=\left(\mathbb{C}^{3}, \omega_{\text {st }}\right)$ and

$$
W_{2}=\left\{(x, y, z, w) \in \mathbb{C}^{4}: x+x^{2} y+z^{2}+w^{3}=0\right\},
$$

where $W_{2}$ inherits the symplectic structure from $\left(\mathbb{C}^{4}, \omega_{\text {st }}\right)$. It can be shown that as smooth manifolds $W_{2} \cong \mathbb{R}^{6}$, hence $W_{1} \cong W_{2}$ smoothly. In contrast, $W_{1} \nRightarrow W_{2}$ as affine algebraic varieties. (This $W_{2}$ is an algebraically exotic structure on $\mathbb{C}^{3}$, known as the Koras-Russell cubic. Being algebraic isomorphic implies being symplectic isomorphic, but the 
converse may fail.) Now we can study the question: are $W_{1}$ and $W_{2}$ isomorphic as symplectic manifolds? That is, does there exist a diffeomorphism $f: W_{1} \rightarrow W_{2}$ such that $f^{*}\left(\omega_{2}\right)=\omega_{1}$ ?

Here is a variation on Problem 1: consider $\mathcal{C}_{m}=$ $\left\{(x, y, z, w) \in \mathbb{C}^{4}: x^{m} y+z w=1\right\}$. As smooth manifolds $\mathcal{C}_{m} \cong \mathbb{S}^{3} \times \mathbb{R}^{3}$ but, for instance, $\mathcal{C}_{1} \not \mathcal{C}_{2}$ are algebraically distinct. Are they symplectically isomorphic? Both versions of this problem will be solved using Legendrian handlebodies, Legendrian wavefronts, and Theorems 1 and 2.

Problem 2 (Propagation of singularities). Consider a planar wavefront $\pi \subseteq \mathbb{R}^{2}$ in the shape of an ellipse moving inwards. Imagine an elliptical source of light, or a elliptical water wave ${ }^{11}$ propagating inwards, as depicted in Figure 6 (upper-left). As wavefronts evolve in time, they develop singularities and it is interesting to understand which singularities are created and how they propagate. ${ }^{12}$ For instance, the Four Cusps Theorem states that, generically, any sequence of wavefronts that starts and ends as in Figure 6 must have a wavefront in the middle with at least four cusps.

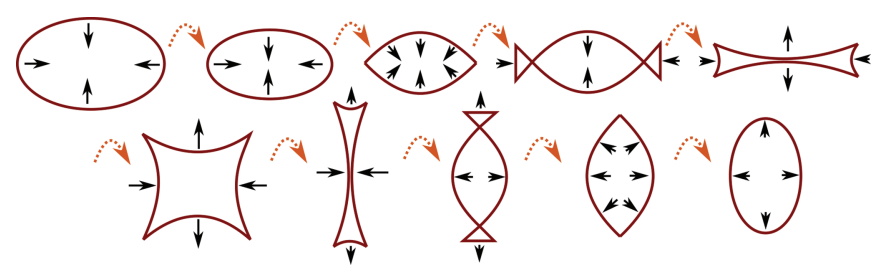

Figure 6. An eversion of an elliptic wavefront. Fixing the first and last fronts, we can ask which and how many singularities must occur for any sequence of intermediate fronts.

In general, given two (possibly singular) wavefronts $\pi_{1}, \pi_{2} \subseteq M$ in a smooth manifold, one can ask which (and how many) singularities must appear or persist for any (generic) sequence of fronts starting at $\pi_{1}$ and ending at $\pi_{2}$. The invariant $\operatorname{Sh}_{\Lambda}(M)$, which studies sheaves constrained by a wavefront $\pi(\Lambda)$, will allow us to answer some such questions.

\section{Legendrian Handlebodies}

Consider the situation in Figure 5, focusing on a Legendrian sphere $\Lambda \subseteq(\partial W, \xi)$ in the contact boundary.

From Legendrians to contact and symplectic. We can use this Legendrian $\Lambda \subseteq \partial W$ to construct a new symplectic manifold $W(\Lambda):=W \cup_{\Lambda} T^{*} \mathbb{D}^{n}$, where $T^{*} \mathbb{D}^{n}$ denotes the (unit disk) cotangent bundle of $\mathbb{D}^{n}$. This $W(\Lambda)$ is obtained by attaching the symplectic piece $T^{*} \mathbb{D}^{n}=\mathbb{D}^{n} \times \mathbb{D}^{n}$, called a

\footnotetext{
${ }^{11}$ These are rare in the ocean, but common in round kiddie pools, fish farms, or a cup of tea.

${ }^{12}$ These problems already appear in classical geometric optics; this is the theory of caustics and wavefronts, key to understanding oscillatory integrals.
}

handle, to (a neighborhood of) the Legendrian $\Lambda$ along its boundary $\partial \mathbb{D}^{n} \times\{0\} .{ }^{13}$ For the appropriate choice of framing (cf. $[6,20])$, A. Weinstein showed that $W(\Lambda)$ admits a symplectic structure $[20]$ and its boundary $Y_{-1}(\Lambda):=\partial(W(\Lambda))$ is a contact manifold [9].

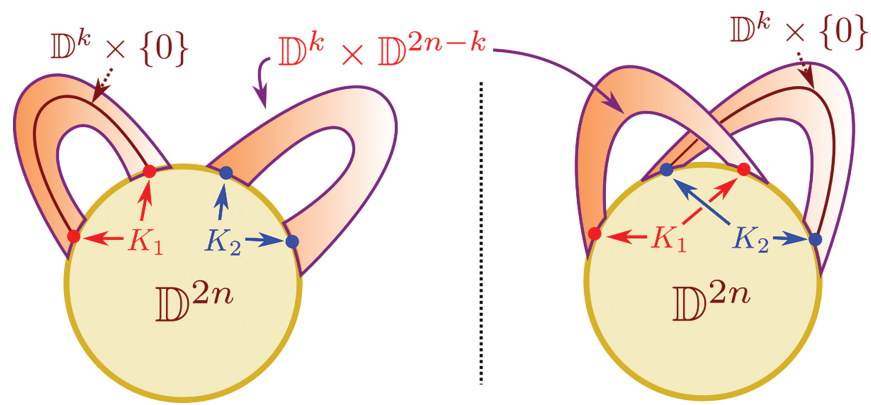

Figure 7. The handle attachment construction of symplectic manifolds: the cores $\mathbb{D}^{k} \times\{0\}$ of the handles $T^{*} \mathbb{D}^{k} \times T^{*} \mathbb{D}^{n-k}$ are attached along (open neighborhoods of) the isotropic spheres $K_{1}, K_{2} \cong \mathbb{S}^{k-1} \subseteq \partial \mathbb{D}^{2 n}$. Differing spheres $K_{1}, K_{2}$ may lead to different contact and symplectic structures.

Given a $k$-dimensional isotropic sphere $S \subseteq(\partial W, \xi)$, we can similarly construct a new symplectic manifold $W(S)$ by attaching the handle $T^{*} \mathbb{D}^{k} \times T^{*} \mathbb{D}^{n-k}$ along its boundary $\partial \mathbb{D}^{k} \times\{0\}$ to $S$. The contact boundary of $W(S)$ is denoted $Y_{-1}(S)$. In either case, the pieces we attach are simple, as they are standard symplectic disks $\left(\mathbb{D}^{2 n}, \omega_{\text {st }}\right)$. The data that most enriches this construction is the choice of where the piece is attached, which we specify by choosing an isotropic submanifold. Intuitively, the wealth of Legendrian submanifolds which are smoothly isomorphic (i.e., smoothly isotopic), but distinct as Legendrians, accounts for the additional richness of contact and symplectic topology, in comparison to differential topology.

Remark. The above construction is a symplectic incarnation of handlebody decompositions for smooth manifolds; see Figure 8 . The advantage of this technique-exemplified by 4 -dimensional Kirby calculus-is that it translates problems about smooth manifolds into (a generalization of) knot theory, where pictorial and combinatorial techniques can be successfully used to manipulate diagrams.

A key distinction is that isotropic submanifolds of $\operatorname{dim}(S) \leq n-2$ are significantly different than Legendrian submanifolds, i.e., those with $\operatorname{dim}(S)=n-1$.

Theorem 4. Let $S_{1}, S_{2} \subseteq(Y, \xi)$ be isotropic, $\operatorname{dim}\left(S_{i}\right)<n-1$, and $S_{1}$ smoothly isotopic ${ }^{14}$ to $S_{2}$. Then $S_{1}$ is isotopic to $S_{2}$, i.e., there exists a 1-parameter family $S_{t}$ of isotropic submanifolds,

\footnotetext{
${ }^{13}$ The second factor of $\mathbb{D}^{n} \times \mathbb{D}^{n}$ is written in a smaller font to emphasize that its only purpose is to thicken $\mathbb{D}^{n}$ to the necessary dimension $2 n$, and make the total space symplectic.

${ }^{14}$ For experts, it should read "formal isotropic isotopic" [6], as we use "smoothly isotopic" for "formally isotopic."
} 


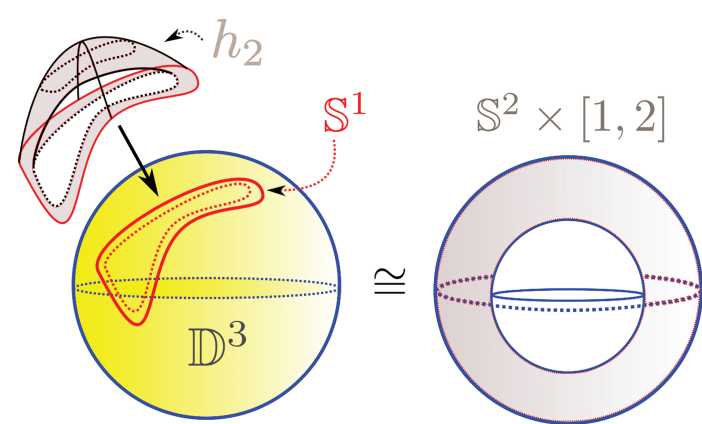

Figure 8. The space $\mathbb{S}^{2} \times[1,2]$ constructed by attaching a 3-dimensional 2-handle $h_{2}$ (an igloo) to a yellow solid 3-disk. The attachment is specified by the red circle in $\partial \mathbb{D}^{3}$.

$t \in[1,2]$, starting at $S_{1}$ and ending at $S_{2}$. In contrast, there exist pairs of Legendrian submanifolds $\Lambda_{1}, \Lambda_{2} \subseteq(Y, \xi)$ which are smoothly isotopic but not Legendrian isotopic.

The message of Theorem 4 is that when we encode contact and symplectic structures in terms of isotropic submanifolds, only those of largest dimension, i.e., Legendrian submanifolds, contain any information beyond smooth topology. In the statement, $\Lambda_{1}, \Lambda_{2}$ are Legendrian isotopic if there exists a 1-parameter family $\Lambda_{t}$ of Legendrian submanifolds, $t \in[1,2]$, starting at $\Lambda_{1}$ and ending at $\Lambda_{2}$. Let us formalize the above construction.

Definition 3. (i) Let $(W, \omega)$ be a symplectic manifold. A Legendrian handlebody is a decomposition of the form $W_{0}=\mathbb{D}^{2 n} \subseteq W_{1} \subseteq \cdots \subseteq W_{S-1} \subseteq$ $W_{s}=W$, where $W_{i+1}=W_{i}\left(\Lambda_{i}\right)$ and $\Lambda_{i} \subseteq\left(\partial W_{i}, \xi_{i}\right)$ is a Legendrian (or isotropic) sphere $[6,20]$.

(ii) Let $(Y, \xi)$ be a contact manifold. A surgery diagram is a sequence $\left(Y^{0}, Y^{1}, \ldots, Y^{s-1}, Y^{s}\right), Y^{0}=$ $\mathbb{S}^{2 n-1}, Y^{S}=Y$, where $Y^{i+1}=Y_{ \pm 1}^{i}\left(\Lambda_{i}\right)$ and $\Lambda_{i} \subseteq\left(Y^{i}, \xi_{i}\right)$ is a Legendrian sphere $[9,16]$ (or an isotropic sphere if doing $Y_{-1}^{i}\left(\Lambda_{i}\right)$ ).

The contact manifold $Y_{+1}(\Lambda)$ is the lower boundary of the (symplectic) manifold $(Y \times[0,1]) \cup_{\Lambda} T^{*} \mathbb{D}^{n}$. This latter manifold is obtained by attaching a $T^{*} \mathbb{D}^{n}$ symplectic handle, with boundary the Legendrian sphere $\Lambda$, to the lower boundary of the symplectic neighborhood $Y \times[0,1]$.

From contact and symplectic to Legendrian. An advantage of these decompositions is generality.

Theorem 5 ([10]). Any closed symplectic manifold $(X, \omega)$ admits a codimension-2 symplectic submanifold $\left(C,\left.\omega\right|_{C}\right) \subseteq(X, \omega)$ such that $X \backslash C$ admits a Legendrian handlebody decomposition.

Thus, proceeding inductively, we assume $\left(C,\left.\omega\right|_{C}\right)$ is understood and study any symplectic manifold $(X, \omega)$ by studying the Legendrian submanifolds that describe $X \backslash C$. Similarly, many interesting contact manifolds $(Y, \xi)$ can be realized as the boundary of $\left(\mathbb{D}^{2 n}, d \lambda_{\mathrm{st}}\right)$ after having either attached or deleted symplectic handles along Legendrian spheres in $\left(\partial \mathbb{D}^{2 n-1}, \operatorname{ker} \lambda_{\text {st }}\right)[9,16]$. Legendrian submanifolds can then be studied using diagrammatic calculus for their wavefronts.

\section{Flexibility: Recent Developments}

Let us address Theorem 1, which pinpoints geometric properties for $X_{1}, X_{2}$ that suffice to conclude isomorphisms $X_{1} \cong X_{2}$. Legendrians being the central character, we start with the following definition.

Definition 4. A Legendrian $\Lambda \subseteq(Y, \xi)$ is said to be stabilized if there exist a point $p \in \Lambda$ and an open neighborhood $\mathcal{O} p(p)=\left(\mathbb{R}^{2 n-1}, \xi_{\mathrm{st}}\right)$, such that its wavefront $\pi(\Lambda) \subseteq \mathbb{R}^{n}$ looks like $Z \times \mathbb{S}^{n-2}$, where $Z$ is the planar zigzag in Figure 9(i). In the lowest dimension $n=2$, a Legendrian link with one zig-zag $Z$, instead of two disjoint zig-zags $Z \times \mathbb{S}^{0}$, is also considered stabilized. (i)

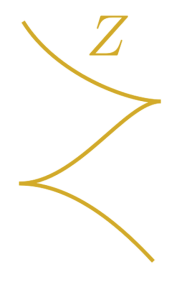

(ii)

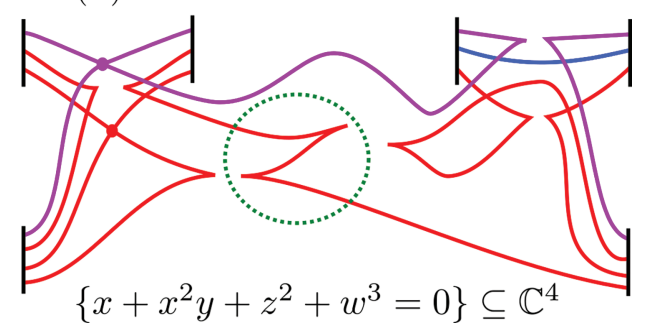

Figure 9. The zig-zag (left). A slice of a Legendrian wavefront handlebody for the Koras-Russell cubic $W_{2}$, with its zig-zag emphasized in the dotted green circle (right).

Any smooth submanifold $N^{n-1} \subseteq(Y, \xi)$ can be approximated by a stabilized Legendrian. In contrast, the Legendrian 2-sphere whose wavefront is drawn in Figure 2 is not stabilized. A reason stabilized Legendrians are useful is the following result, which establishes them as flexible objects with respect to the property of being stabilized (and smoothly isotopic).

Theorem 6. Let $\Lambda_{1}, \Lambda_{2} \subseteq(Y, \xi)$ be smoothly isotopic and each of them (sufficiently) stabilized. Then there exists a Legendrian isotopy from $\Lambda_{1}$ to $\Lambda_{2}$.

For $n=2$, this is a theorem of D. Fuchs and S. Tabachnikov (1997) and the sufficiently hypothesis is required; that is, there must exist sufficiently many points $p \in Y$ with a zig-zag near them. For higher dimensions $n \geq 3$, this is the result of E. Murphy's thesis (2012), and the adverb sufficiently is not needed: one zig-zag is enough. The stabilization procedure used in Theorem 6 was introduced by Ekholm-Etnyre-Sullivan (2005).

The strength of this result is that, in general, the existence of a smooth isotopy is not enough to conclude a Legendrian isotopy. Nevertheless, if we draw $\Lambda_{1}$ and $\Lambda_{2}$ and we see zig-zags in their fronts, then we can conclude that there is a Legendrian isotopy. 
An analogue of Definition 4 for contact and symplectic manifolds uses the Legendrian handlebodies and surgery diagrams introduced above.

Definition 5. A symplectic structure $(W, d \lambda)$ is said to be flexible if it admits a Legendrian handlebody whose Legendrians are all stabilized. ${ }^{15}$

A contact structure is overtwisted if it admits a contact surgery diagram where a handle attached along a stabilized Legendrian has been deleted, i.e., $Y=Y_{+1}(\Lambda)$ for $\Lambda$ a stabilized Legendrian.

It is proven in [5] that this definition of overtwisted contact structures coincides with the classical definition of Y. Eliashberg (1989). In line with Theorem 6, we know uniqueness for these flexible objects, i.e., their diffeomorphism type and being stabilized completely characterizes their contact and symplectic geometry.

Theorem 7. Let $\left(W_{1}, d \lambda_{1}\right)$ and $\left(W_{2}, d \lambda_{2}\right)$ be two flexible symplectic structures and $W_{1}$ diffeomorphic ${ }^{16}$ to $W_{2}$. Then $W_{1}$ is symplectomorphic to $W_{2}$. Similarly, let $\left(Y_{1}, \xi_{1}\right)$ and $\left(Y_{2}, \xi_{2}\right)$ be overtwisted contact structures and $Y_{1}$ diffeomorphic to $Y_{2}$. Then $\left(Y_{1}, \xi_{1}\right)$ is contact isomorphic to $\left(Y_{2}, \xi_{2}\right)$.

Fix a smooth manifold. Theorem 7 states that, should either of these structures exist-flexible symplectic or overtwisted contact-then they are unique. We also emphasize that the general existence of contact structures has also been proved.

Theorem 8 ([2]). Let $Y^{2 n-1}$ be a smooth manifold whose tangent bundle $T Y$ is an almost-complex vector bundle. Then $Y$ admits a (overtwisted) contact structure.

Solution to Problem 1. The symplectic manifold $W_{1}=\mathbb{C}^{3}$ is tautologically flexible, as it admits the empty handlebody $W_{1}=\mathbb{D}^{6}(\emptyset)$. To study $W_{2}$, we use the Legendrian handlebody $W_{2}=\mathbb{D}^{6}(\Lambda)$, where $\Lambda$ is the Legendrian surface whose (sliced) wavefront is drawn in Figure 9. Since this $\Lambda$ is stabilized and $W_{2}$ is smoothly identical to $W_{1}$, Theorem 7 implies that $W_{1}$ is symplectomorphic to $W_{2}$. In fact, this same argument shows that $W_{1}$ is Stein deformation equivalent to $W_{2}[6]$.

Partial solution to Variation 1. The wavefronts of the Legendrian handlebodies for $\mathcal{C}_{m}$ are drawn in Figure 10. Since they have zig-zags, this proves that $\mathcal{C}_{m}$ are flexible for $m \geq 2$ and thus-by Theorem $7-\mathcal{C}_{l}$ is symplectomorphic (and even Stein equivalent) to $\mathcal{C}_{m}$ for any $l, m \geq 2$.

\footnotetext{
${ }^{15}$ Each Legendrian component has to admit a zig-zag in the complement of the other Legendrians.

${ }^{16}$ Recall that we use the term diffeomorphic to also mean that the formal obstructions distinguishing $W_{1}, W_{2}$ vanish.
}

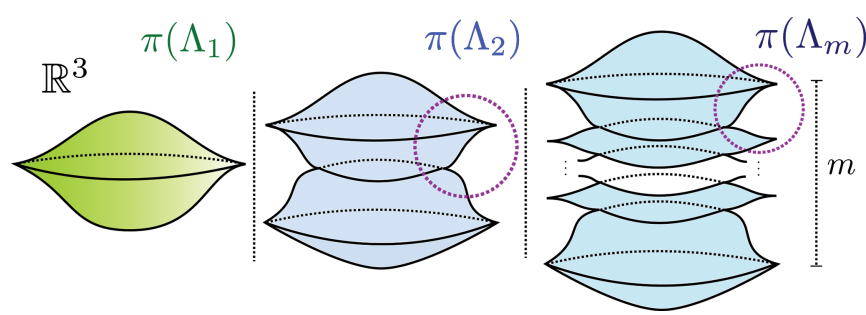

Figure 10. The wavefronts $\pi\left(\Lambda_{m}\right)$ for the symplectic manifolds $\mathcal{C}_{m}=\mathbb{D}^{6}\left(\Lambda_{m}\right)$.

Note that the front for $\mathcal{C}_{1}=\mathbb{D}^{6}\left(\Lambda_{1}\right)$ does not readily exhibit a zig-zag: to completely resolve Variation 1, we either manipulate $\Lambda_{1}$ until a zig-zag appears ${ }^{17}$ or, if we believe $\Lambda_{1}$ is not equal to the other $\Lambda_{m}$, use an invariant such that $I\left(\Lambda_{1}\right) \neq I\left(\Lambda_{m}\right), m \geq 2$. This matter is resolved in the next section. (It turns out that the latter is correct, so an invariant is needed.)

\section{Rigidity: New Invariants and Results}

Let us consider a Legendrian $\Lambda \subseteq\left(\mathbb{R}^{2 n-1}, \xi_{\text {st }}\right)$ : we would like to come up with an invariant $I(\Lambda)$, i.e., $I\left(\Lambda_{1}\right)=I\left(\Lambda_{2}\right)$ if $\Lambda_{1}, \Lambda_{2}$ are Legendrian isotopic. ${ }^{18}$ In addition, we want this invariant $I(\Lambda)$ to be effective, in that it is often able to tell apart distinct pairs of Legendrians $\Lambda_{1}, \Lambda_{2} \subseteq\left(\mathbb{R}^{2 n-1}, \xi_{\text {st }}\right)$ which are smoothly equal. Thanks to studying the wavefront projection $\pi(\Lambda) \subseteq \mathbb{R}^{n}$, such a new invariant has been constructed and studied recently.

Theorem $9([13,14,18])$. The category $\operatorname{Sh}_{\Lambda}\left(\mathbb{R}^{n}\right)$ of constructible sheaves microlocally supported at the Legendrian $\Lambda$ is a Legendrian invariant. In addition, it is effective and can be computed in many cases.

Let us informally explain why we end up considering such invariant $I(\Lambda):=\operatorname{Sh}_{\Lambda}\left(\mathbb{R}^{n}\right)$ :

(1) The wavefront $\pi(\Lambda)$ gives a stratification $\mathcal{S}(\pi(\Lambda))$ of $\mathbb{R}^{n}$. The singularities of $\pi(\Lambda)$ are responsible for the richness of these stratifications; ${ }_{i}^{19}$ we can then apply techniques to study stratified spaces, such as constructible sheaves. ${ }^{20}$

(2) A Legendrian $\Lambda$ is recovered from its wavefront $\pi(\Lambda)$ by taking the first partial derivatives: we capture the information about the first derivatives with the notion of microlocal support $\mu \operatorname{supp}(F) \subseteq$ $T^{\infty} \mathbb{R}^{n}$ of a sheaf $F$.

Intuitively, $\mu \operatorname{supp}(F) \subseteq T^{*} \mathbb{R}^{n}$ encodes points $p \in \mathbb{R}^{n}$ and (unit) directions $v \in T_{p} \mathbb{R}^{n}$ such that there are

\footnotetext{
${ }^{17}$ This would prove $\Lambda_{1}$ is stabilized.

${ }^{18}$ The next paragraphs can be generalized to $\mathrm{Sh}_{\Lambda}(M)$ for a smooth manifold $M$ and $\Lambda \subseteq T^{\infty} M$ a Legendrian submanifold of the unit cotangent bundle $T^{\infty} M$.

${ }^{19}$ If $\pi(\Lambda)$ were smooth, there would only be two strata: $\pi(\Lambda)$ and $\mathbb{R}^{n} \backslash \pi(\Lambda)$ Intuitively, the invariant in this case is given by the derived category of local systems on $\mathbb{R}^{n} \backslash \pi(\Lambda)$.

${ }^{20}$ The classical stratified theory developed notions such as stratified Morse theory, perversity, and intersection homology.
} 
sections of $F$ which cannot be extended as we move from $p$ in the direction $v$. This is a sheaf-theoretic analogue, due to Kashiwara-Schapira (1982), of Hörmander's wavefront set of a function (1971). The category $\operatorname{Sh}_{\Lambda}\left(\mathbb{R}^{n}\right)$ is then defined as the (dg-derived) category of sheaves $F$ on $\mathbb{R}^{n}$ which are both constructible with respect to $\mathcal{S}(\pi(\Lambda))$ and satisfy $\mu \operatorname{supp}(F) \subseteq \Lambda$. In many instances, this category can be computed combinatorially, by assigning a local system to each stratum of $\mathcal{S}(\pi(\Lambda))$ and maps between these local systems.

Solution to Variation 1. Consider the wavefronts $\pi\left(\Lambda_{1}\right)$ and $\pi\left(\Lambda_{m}\right), m \geq 2$, in Figure 10. The category $I\left(\Lambda_{\eta}\right):=$ $\mathrm{Sh}_{\Lambda_{\eta}}\left(\mathbb{R}^{3}\right)$ has at least one object for $\eta=1$ and none for $\eta=m$. By Theorem 9, $\Lambda_{1}$ is not Legendrian isotopic to $\Lambda_{m}$. This Legendrian wavefront description of the Stein manifolds $\mathcal{C}_{n}$ also implies that $\mathcal{C}_{1}$ is not symplectomorphic to $\mathcal{C}_{m}, m \geq 2$.

Solution to Problem 2. The category $\mathrm{Sh}_{\Lambda}\left(\mathbb{R}^{n}\right)$ also knows about certain geometric properties for all possible wavefronts $\pi(\Lambda)$ of a Legendrian. This is beautifully used by S. Guillermou ${ }^{21}$ to prove some of Arnol'd's Cusps Conjectures [13].

Theorem 10. (4 Cusps) A generic family of wavefronts starting and ending with the same wavefronts as in Figure 6 must have an intermediate wavefront with at least four cusps.

(3 Cusps) Any generic wavefront deformation of a cotangent fiber in $\mathbb{R P}^{2}$ must have at least three cusps.

The proof of the 3 Cusps Conjecture [13] studies the category $\mathrm{Sh}_{\Lambda_{0}}\left(\mathbb{S}^{2}\right)$ for the initial point wavefront $\Lambda_{i}$, which has countably many "simple" objects. Then it is argued that the category $\operatorname{Sh}_{\Lambda_{f}}\left(\mathbb{S}^{2}\right)$ associated to any generic wavefront deformation $\Lambda_{f}$ with only one cusp must have uncountably many such objects. Since $\mathrm{Sh}_{\Lambda_{f}}\left(\mathbb{S}^{2}\right)$ should be an invariant under deformation of $\Lambda_{i}$, and the number of cusps is odd, there must be at least three cusps.

Take-home nugget. We can describe contact $(Y, \xi)$ and symplectic $(W, \omega)$ manifolds with Legendrian submanifolds $\Lambda$ via Legendrian handlebodies and surgeries. Legendrians can themselves be studied using a diagrammatic calculus for their wavefronts: they are completely classified if they have a zig-zag in their wavefront, and else we have computable invariants $I(\Lambda)$ which both distinguish them and tell us about their geometric properties. These Legendrian invariants $I(\Lambda)$ can in turn be used to provide invariants of contact and symplectic manifolds $(Y, \xi)$ and $(W, \omega)$ and help their classification.

Sins of omission. Floer theory and the study of (pseudo)holomorphic curves are also pillars of modern

\footnotetext{
${ }^{21}$ The 4 Cusps Conjecture was previously proved by Chekanov-Pushkar (2005) by different means.
}

contact and symplectic topology. They have been successfully applied to obtain groundbreaking results for the last three decades, preceding the developments presented here. There is a (currently) conjectural relation between Floertheoretical and sheaf-theoretical invariants [8]. ${ }^{22}$

Finally, a few examples of other recent developments in the field are: results towards "2-or- $\infty$ many Reeb orbits," by M. Hutchings et al., the resolution of the Simplicity Conjecture [7] in $C^{0}$-symplectic geometry, ${ }^{23}$ and the use of stability conditions in the study of symplectic mapping class groups and auto-equivalences of Fukaya categories, after I. Smith et al. Equally interesting, the construction of infinitely many Lagrangian tori by D. Auroux and R. Vianna, the development of arboreal Lagrangian skeleta [19] after D. Nadler, L. Starkston, et al., and the study of Liouville sectors and their invariants [8].

ACKNOWLEDGMENTS. I warmly thank H. Gao,

L. Heuberger, S. Ogami, S. Rubin, J. S. Rodríguez, M. Vazirani, A. Waldron, and the referees.

\section{References}

[1] V. I. Arnol'd, Singularities of caustics and wave fronts, Mathematics and its Applications (Soviet Series), vol. 62, Kluwer Academic Publishers Group, Dordrecht, 1990. MR1151185

[2] Matthew Strom Borman, Yakov Eliashberg, and Emmy Murphy, Existence and classification of overtwisted contact structures in all dimensions, Acta Math. 215 (2015), no. 2, 281-361, DOI 10.1007/s11511-016-0134-4. MR3455235

[3] R. Casals and H. Gao, Infinitely many Lagrangian fillings, arXiv, 2020.

[4] Roger Casals and Emmy Murphy, Legendrian fronts for affine varieties, Duke Math. J. 168 (2019), no. 2, 225-323, DOI 10.1215/00127094-2018-0055. MR3909897

[5] Roger Casals, Emmy Murphy, and Francisco Presas, Geometric criteria for overtwistedness, J. Amer. Math. Soc. 32 (2019), no. 2, 563-604, DOI 10.1090/jams/917. MR3904160

[6] Kai Cieliebak and Yakov Eliashberg, From Stein to Weinstein and back: Symplectic geometry of affine complex manifolds, American Mathematical Society Colloquium Publications, vol. 59, American Mathematical Society, Providence, RI, 2012. MR3012475

[7] D. Cristofaro-Gardiner, V. Humilière, and S. Seyfaddini, Proof of the simplicity conjecture, arXiv, 2020.

[8] Sheel Ganatra, John Pardon, and Vivek Shende, Covariantly functorial wrapped Floer theory on Liouville sectors, Publ. Math. Inst. Hautes Études Sci. 131 (2020), 73-200, DOI 10.1007/s10240-019-00112-x. MR4106794

[9] Hansjörg Geiges, An introduction to contact topology, Cambridge Studies in Advanced Mathematics, vol. 109, Cambridge University Press, Cambridge, 2008. MR2397738

\footnotetext{
${ }^{22}$ Informally, it might be possible that they contain the same information. Nevertheless, there are several central results which are proven with one method, and yet a proof using the other method is still to be found. Exciting times ahead.

${ }^{23}$ The group of area-preserving homeomorphisms of the 2-disk is not simple.
} 


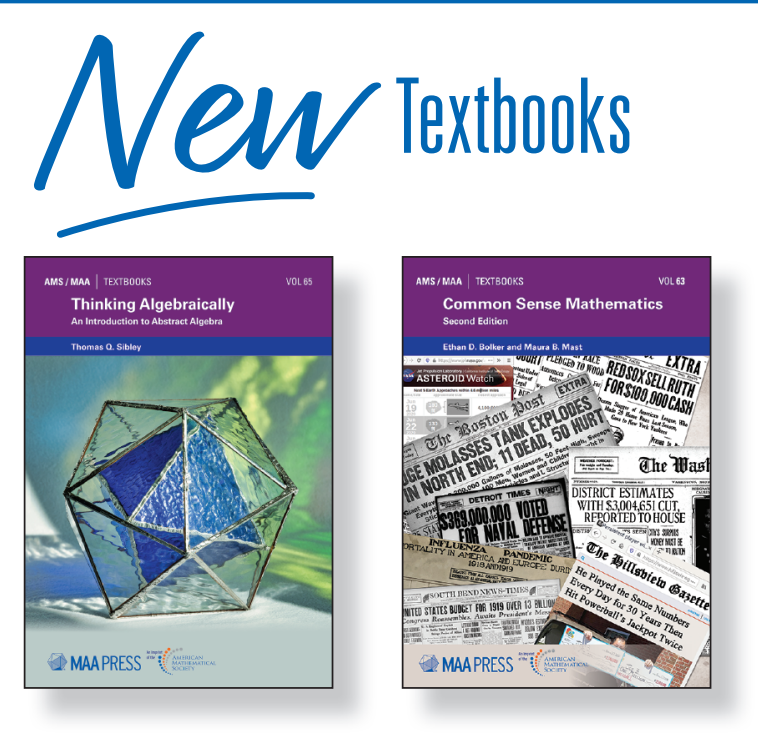

Thinking Algebraically

\section{An Introduction to Abstract Algebra}

AMS / MAA Press

Thomas Q. Sibley, St. John's University, Collegeville, $\mathrm{MN}$

Thinking Algebraically presents the insights of abstract algebra in a welcoming and accessible way. It succeeds in combining the advantages of rings-first and groups-first approaches while avoiding the disadvantages.

AMS/MAA Textbooks, Volume 65; 2021; 592 pages; Softcover; ISBN: 978-1-4704-6030-3; List US\$85; Individual member US\$63.75; MAA members US\$63.75; Order codeTEXT/65

\section{Common Sense Mathematics}

\section{Second Edition AMs / MAA Press}

Ethan D. Bolker, University of Massachusetts Boston, MA, and Maura B. Mast, Fordham University, Bronx, NY

This newly revised text helps students to think carefully-and critically-about numerical information in everyday contexts.

AMS/MAA Textbooks, Volume 63; 2021; 342 pages; Softcover; ISBN: 978-1-4704-6134-8; List US\$75; Individual member US\$56.25; MAA members US\$56.25; Order code TEXT/63

\section{=Textbook Visit bookstore.ams.org}

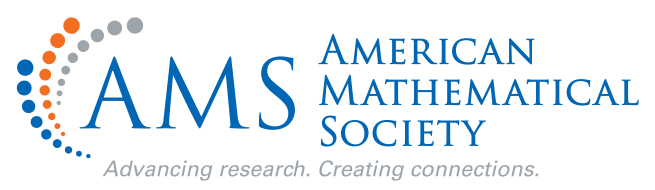

[10] Emmanuel Giroux, Remarks on Donaldson's symplectic submanifolds, Pure Appl. Math. Q. 13 (2017), no. 3, 369-388, DOI 10.4310/pamq.2017.v13.n3.a1. MR3882202

[11] Robert E. Gompf, Handlebody construction of Stein surfaces, Ann. of Math. (2) 148 (1998), no. 2, 619-693, DOI 10.2307/121005. MR1668563

[12] Mikhael Gromov, Partial differential relations, Ergebnisse der Mathematik und ihrer Grenzgebiete (3) [Results in Mathematics and Related Areas (3)], vol. 9, SpringerVerlag, Berlin, 1986. MR864505

[13] S. Guillermou, Sheaves and symplectic geometry of cotangent bundles, arXiv, 2019.

[14] Stéphane Guillermou, Masaki Kashiwara, and Pierre Schapira, Sheaf quantization of Hamiltonian isotopies and applications to nondisplaceability problems, Duke Math. J. 161 (2012), no. 2, 201-245, DOI 10.1215/00127094-1507367 MR2876930

[15] Stéphane Guillermou and Pierre Schapira, Microlocal theory of sheaves and Tamarkin's non displaceability theorem, Homological mirror symmetry and tropical geometry, Lect. Notes Unione Mat. Ital., vol. 15, Springer, Cham, 2014, pp. 43-85, DOI 10.1007/978-3-319-065144_3. MR3330785

[16] Burak Ozbagci and András I. Stipsicz, Surgery on contact 3-manifolds and Stein surfaces, Bolyai Society Mathematical Studies, vol. 13, Springer-Verlag, Berlin; János Bolyai Mathematical Society, Budapest, 2004. MR2114165

[17] Vivek Shende, David Treumann, Harold Williams, and Eric Zaslow, Cluster varieties from Legendrian knots, Duke Math. J. 168 (2019), no. 15, 2801-2871, DOI 10.1215/00127094-2019-0027. MR4017516

[18] Vivek Shende, David Treumann, and Eric Zaslow, Legendrian knots and constructible sheaves, Invent. Math. 207 (2017), no. 3, 1031-1133, DOI 10.1007/s00222-016-06815. MR3608288

[19] Laura Starkston, Arboreal singularities in Weinstein skeleta, Selecta Math. (N.S.) 24 (2018), no. 5, 4105-4140, DOI 10.1007/s00029-018-0441-z. MR3874691

[20] Alan Weinstein, Contact surgery and symplectic handlebodies, Hokkaido Math. J. 20 (1991), no. 2, 241-251, DOI 10.14492/hokmj/1381413841. MR1114405

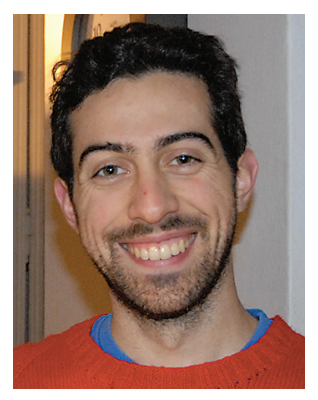

Roger Casals

Credits

Opening image and photo of Roger Casals is courtesy of Carles Casals.

Figures 1-10 are courtesy of Roger Casals. 